\title{
Massive nerve root enlargement in chronic inflammatory demyelinating polyneuropathy
}

\author{
W Schady, P J Goulding, B R F Lecky, R H M King, C M L Smith
}

Walton Centre for

Neurology and

Neurosurgery, Rice

Lane, Liverpool

L9 1AE, UK

B R F Lecky

Royal Free Hospital, Pond Street, London

NW3 2QG, UK

R H M King

Royal Hallamshire

Hospital, Glossop

Road, Sheffield

S10 2JF, UK

C M L Smith

Correspondence to:

Dr W Schady, Department of Neurology, Manchester Royal Infirmary, Manchester M13 9WL, UK

Received 25 August 1995 and in final revised form

7 August 1996

Accepted 20 August 1996

(F Neurol Neurosurg Psychiatry 1996;61:636-640)

Keywords: root hypertrophy; chronic idiopathic demyelinating polyneuropathy

Chronic inflammatory demyelinating polyneuropathy (CIDP) is a relapsing or chronically progressive disorder most commonly presenting with limb weakness, distal sensory disturbance, and hyporeflexia. ${ }^{12}$ The CSF protein is almost always raised. ${ }^{3}$ Nerve conduction studies show slowing of motor conduction and sural nerve biopsy discloses segmental demyelination and remyelination. ${ }^{1-3}$ CIDP is one of the causes of "hypertrophic neuropathy". Thickened peripheral nerves were detected in $11 \%$ of one large

\begin{abstract}
Department of
Neurology,

Manchester Royal

Infirmary,

Manchester, UK

W Schady

P J Goulding

Abstract

Objective-To report three patients with chronic inflammatory demyelinating polyneuropathy (CIDP) presenting with symptoms suggestive of cervical (one patient) and lumbar root disease.

Methods-Nerve conduction studies, EMG, and nerve biopsy were carried out, having found the nerve roots to be very enlarged on MRI, CT myelography, and at surgery.

Results-Clinically, peripheral nerve thickening was slight or absent. Subsequently one patient developed facial nerve hypertrophy. This was mistaken for an inner ear tumour and biopsied, with consequent facial palsy. Neurophysiological tests suggested a demyelinating polyneuropathy. Sural nerve biopsy showed in all cases some loss of myelinated fibres, inflammatory cell infiltration, and a few onion bulbs. Hypertrophic changes were much more prominent on posterior nerve root biopsy in one patient: many fibres were surrounded by several layers of Schwann cell cytoplasm. There was an excellent response to steroids in two patients but not in the third (most advanced) patient, who has benefited only marginally from intravenous immunoglobulin therapy.

Conclusions-MRI of the cauda equina may be a useful adjunct in the diagnosis of CIDP.
\end{abstract}

series $^{1}$ and in up to a third of relapsing cases. ${ }^{4}$ Greatly enlarged nerve roots have been identified in a few typically affected patients at necropsy, ${ }^{56}$ by myelography, ${ }^{7}$ at surgery, ${ }^{8}$ and by MRI. ${ }^{9-11}$ We report three patients who presented with symptoms suggestive of radiculopathy, in whom MRI or CT myelography showed massive nerve root hypertrophy. Subsequent investigation, including nerve root biopsy in one patient, showed CIDP.

\section{Case reports}

CASE 1

A man aged 58 developed severe left groin pain while walking, eased by flexing the limb, with associated low backache and decreased walking speed. He then noticed deadness and increased sensitivity of his right little finger. $\mathrm{He}$ was thought to have a cervical spondylotic radiculomyelopathy, for which a decompressive cervical laminectomy was performed at another hospital in 1985. The clinical and radiological findings at that time are not known. The operation was not helpful and his walking continued to deteriorate. By 1993 his condition had progressively worsened resulting in weakness of his right arm and both legs, sensory disturbance in his hands and in the lower half of his body, and occasional urge incontinence. His balance was poor, especially in the dark, and he was unable to stand without the aid of a stick. There was no family history of polyneuropathy.

Examination showed restricted neck and low back movements. There was profound wasting of the forearms and small hand muscles, worse on the right. His upper limbs were very weak, more so distally, with moderate predominantly proximal asymmetric weakness of the lower limbs. All tendon reflexes were absent but the plantar responses were extensor. The peripheral nerves felt slightly thickened. There was hypersensitivity of the skin in both little fingers and medial forearms and sensory loss from the umbilicus downwards in a patchy distribution. Joint position sense was impaired in the feet and vibration could only be detected above the shoulders. He was unable to stand without support but he could take a few steps with the aid of a stick. Romberg's test was strongly positive. 

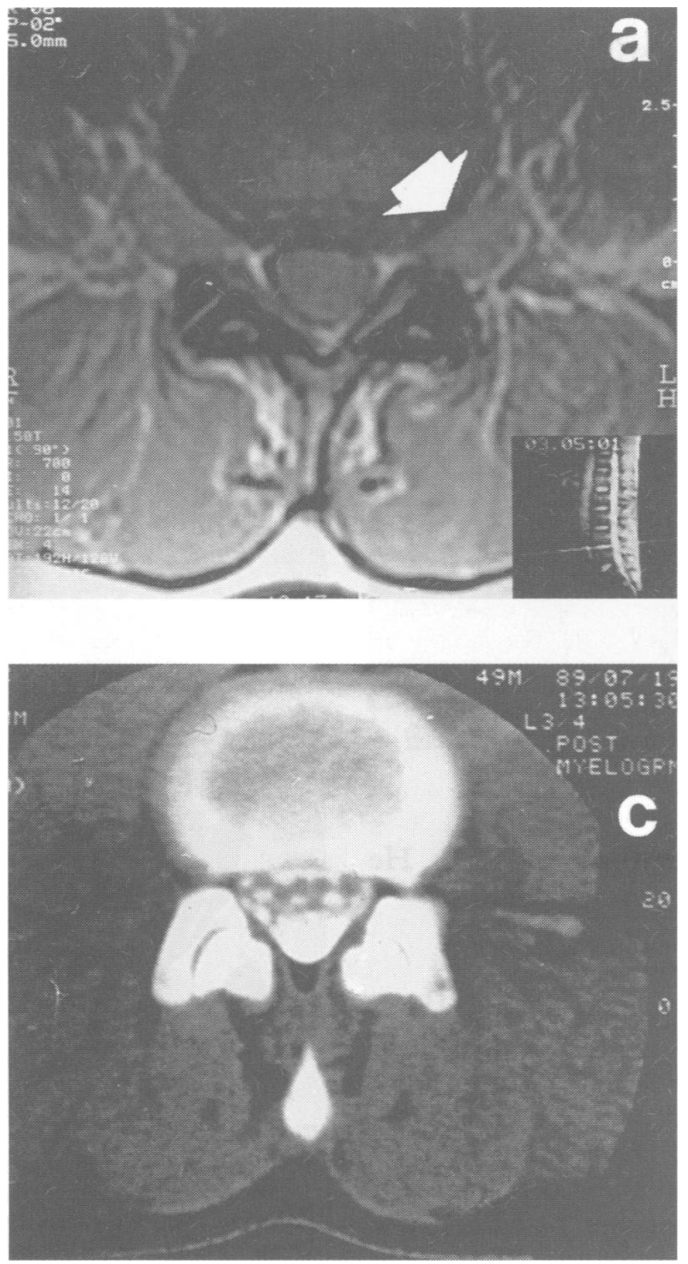

A blood count, erythrocyte sedimentation rate, liver and renal function tests, blood glucose, immunoglobulins, and plasma protein and urine electrophoresis were all normal. A full antibody screen (including MAG antibodies) was negative. Molecular genetic studies disclosed no duplication at $17 \mathrm{p} 11 \cdot 2$. No acid fast bacilli were seen on skin scrapings. Magnetic resonance imaging showed atrophy of the cervical cord. There was striking bilateral enlargement of all cervical nerve roots with widening of the exit foraminae, especially at C6-7 and C7-T1. The thoracic spine, cord, and nerve roots appeared normal. There was homogeneously increased signal from the cauda equina, with multiple areas of nodular enhancement and no visualisation of CSF. The lumbar nerve roots were grossly enlarged, filling and expanding the exit foramina (fig 1a). Nerve conduction studies (table) showed reduced sensory and motor action potentials

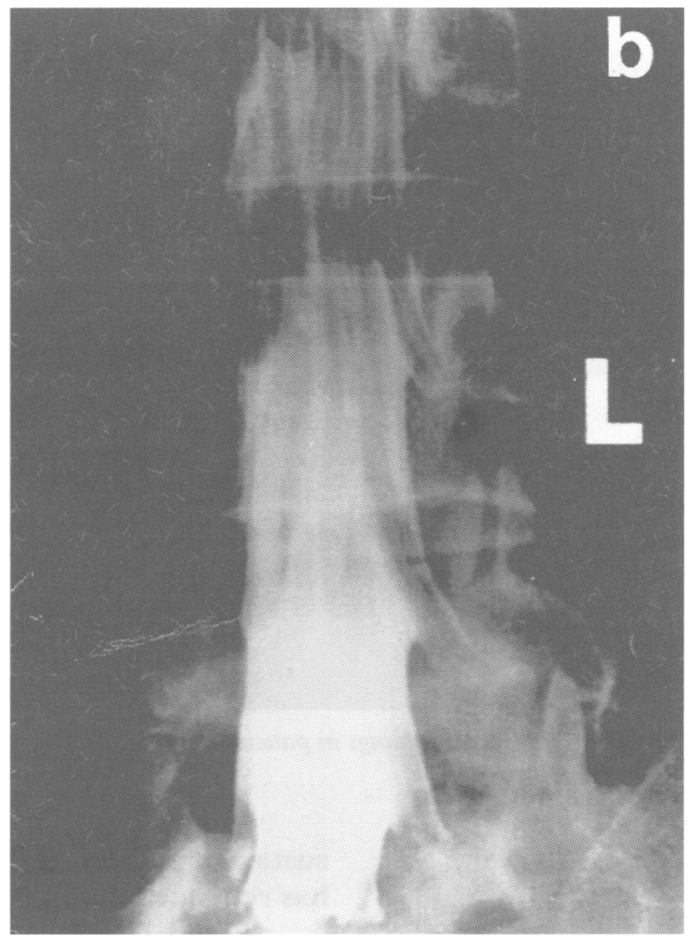

Figure 1 (a) MRI axial T1 weighted gradient echo image, patient 1; (b) myelogram, patient 2; (c) CT myelogram, patient 3 . There is pronounced enlargement of the lumbosacral nerve roots, which in patient 1 (a) are seen to expand in a dumbbell shape outside the exit foramen (arrow).

and slowing of nerve conduction and motor conduction block, in keeping with a demyelinating polyneuropathy.

A lumbar laminectomy was undertaken to decompress the cauda equina. On opening the dura several thickened, white, and matted nerve roots were seen (fig 2). No CSF was obtained. A fascicular biopsy of a posterior nerve root showed loss of myelinated axons and a few endoneurial inflammatory cells. Many large fibres had thin myelin sheaths and were surrounded by several layers of Schwann cell cytoplasm ("onion bulbs"). A sural nerve biopsy disclosed similar but less advanced changes. Unmyelinated axons were normal.

The neurophysiological and pathological findings supported a diagnosis of CIDP. Treatment with prednisolone and azathioprine resulted in initial worsening of hand strength, bladder control, and mobility, such that he became unable to stand unassisted. Pooled intravenous immunoglobulin therapy (Sandoglobulin $(0.4 \mathrm{~g} / \mathrm{kg} /$ day $)$ for five days followed by monthly infusions of $22-24 \mathrm{~g}$ for 10 months) led to mild improvement in hand function (less than 1 MRC grade) but had no

Nerve conduction data

\begin{tabular}{|c|c|c|c|c|c|c|c|c|c|c|c|c|c|c|c|}
\hline \multirow[b]{3}{*}{ Patient } & \multicolumn{12}{|c|}{ Motor } & \multirow{2}{*}{\multicolumn{3}{|c|}{$\frac{\text { Sensory }}{\text { Amplitude }(\mu V)}$}} \\
\hline & \multicolumn{3}{|c|}{ Amplitude $(m V)$} & \multicolumn{3}{|c|}{$\%$ Conduction block } & \multicolumn{3}{|c|}{ Distal latency (ms) } & \multicolumn{3}{|c|}{ Conduction velocity $(\mathrm{m} / \mathrm{s})$} & & & \\
\hline & 1 & 2 & 3 & 1 & 2 & 3 & 1 & 2 & 3 & 1 & 2 & 3 & 1 & 2 & 3 \\
\hline
\end{tabular}

^Lower limb sensory action potentials are for the sural nerve. Compound muscle action potential amplitudes correspond to those obtained on distal nerve stimulation. Sensory action potentials were recorded orthodromically in the upper limbs and antidromically in the lower limbs. \% Conduction block refers to the drop in motor potential amplitude between distal and proximal stimulation sites. 


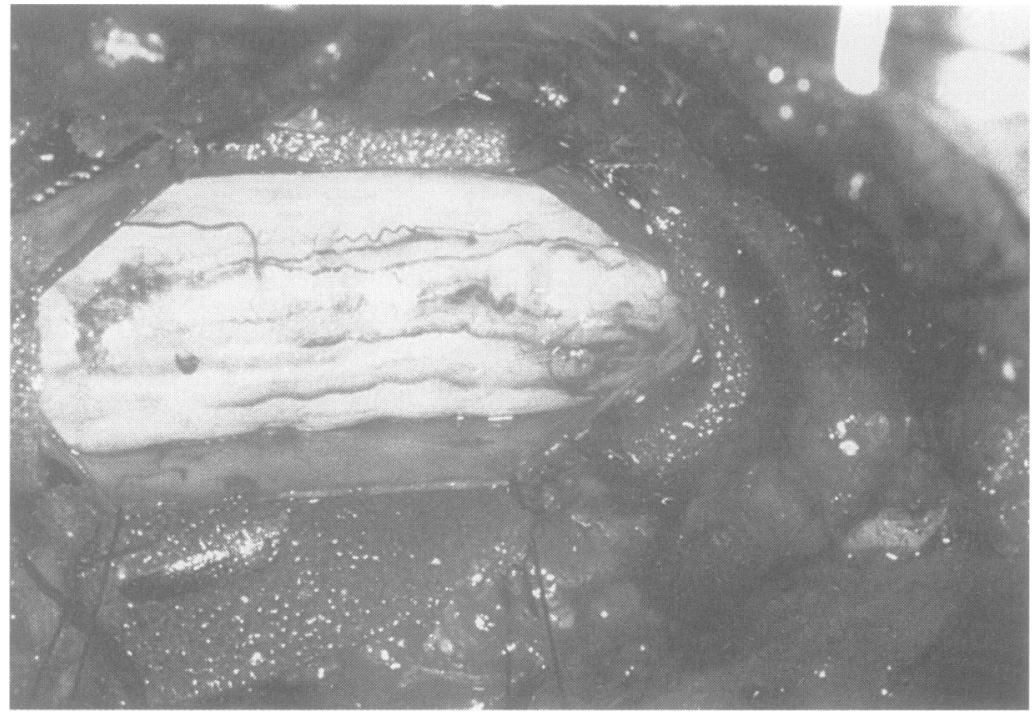

Figure 2 Operative findings in patient 1. The lumbar nerve roots are grossly thickened and matted together.

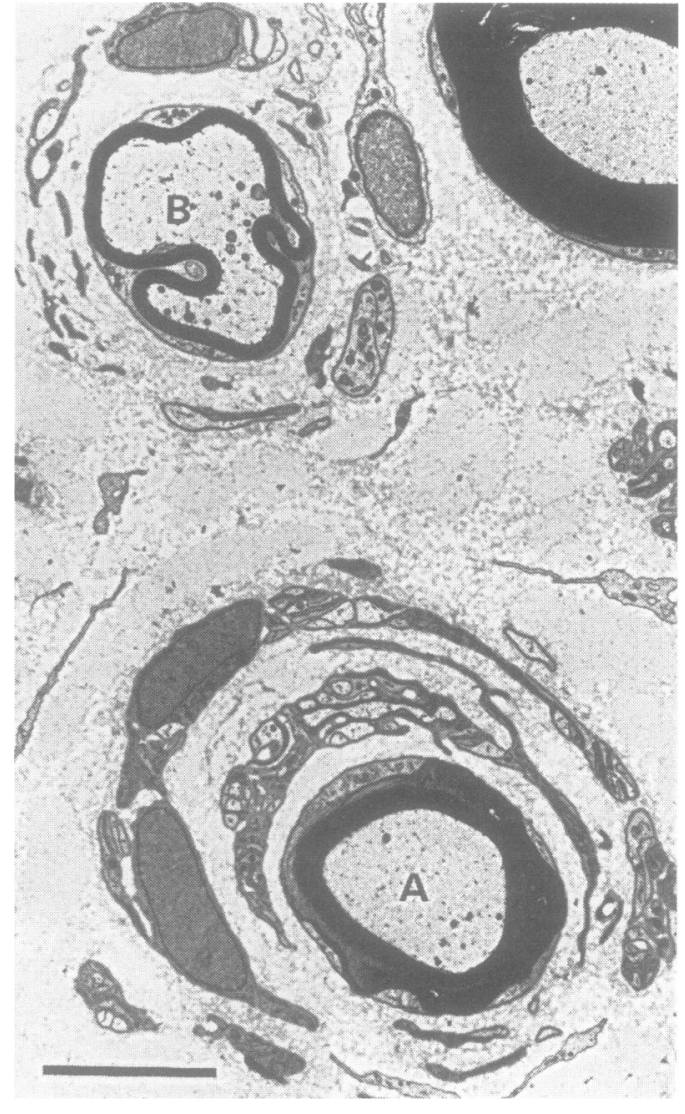

Figure 3 Sural nerve from patient 2 showing an onion bulb consisting of a small, normally myelinated fibre $(A)$ surrounded by two layers of circumferentially oriented Schwann cells. The thinly myelinated fibre (B) is surrounded by a less well defined layer of small Schwann cell profiles. Bar $=5 \mu \mathrm{m}$. but no pain or sensory complaints. The upper limbs were asymptomatic except for occasional cramping of the hands. There was no family history of neuromuscular disease.

Examination showed mild wasting and weakness of the small hand muscles. There was moderately severe symmetric wasting and weakness of the quadriceps and mild weakness of hip flexion, hip extension, and ankle dorsiflexion. The upper limb reflexes were preserved but the knee jerks were sluggish and the ankle jerks were absent. Plantar responses were flexor. There was no sensory deficit except for loss of vibration sense at the toes.

Standard haematological and biochemical tests were normal. Serum creatine kinase concentration was mildly raised at 335-462 U/1 (upper limit of normal $175 \mathrm{U} / 1$ ). There was no abnormal paraprotein. The CSF protein was $1.06 \mathrm{~g} / 1$. Anti-MAG antibodies were not detected and there were no genetic markers for HMSN1. Spina bifida occulta was seen on radiography of the lumbar spine. A lumbar myelogram showed pronounced hypertrophy of all lumbosacral nerve roots (fig 1b). Motor conduction was slowed in the upper and lower limbs (table). On EMG there was evidence of chronic partial denervation in vasti and active denervation in tibialis anterior, with fasciculation. A biopsy of the quadriceps showed mild neurogenic atrophy. A sural nerve biopsy disclosed some loss of myelinated axons and occasional axonal sprouts. A few thinly myelinated fibres were surrounded by whorls of Schwann cell cytoplasm (fig 3). Slight perineurial macrophage infiltration was seen as well as small but definite deposits of both CD4+ and CD8+ cells.

The initial clinical impression was of a lumbar polyradiculopathy, possibly in relation to her spina bifida occulta. However, the results of investigations pointed to CIDP and she was therefore treated with $60 \mathrm{mg}$ prednisolone and $150 \mathrm{mg}$ azathioprine daily. Improvement began within a few weeks and after eight months she was almost asymptomatic. Prednisolone was gradually withdrawn over a year and azathioprine was stopped after three years. Her strength has remained normal.

\section{CASE 3}

A 49 year old man was referred in 1989 because of foot drop. He reported several episodes over the previous 20 years of severe low back pain radiating to the anterior thighs, resolving with conservative treatment. In 1981 he developed pain in his left foot, which was helped by a metatarsal support. Five years later his wife noticed that his left foot slapped down when he walked. The foot drop gradually worsened and in 1988 he became aware of difficulty rising from a squat, and twitching of the thigh muscles. His medical history included surgery to correct a squint in 1948 and 1983. In 1987 he developed a postural tremor which responded to treatment with propranolol. His mother had a similar tremor. There was no family history of a polyneuropa- 
thy and nerve conduction studies in both his children were normal.

Examination of the legs disclosed some wasting of the quadriceps, hamstrings, and extensor digitorum brevis muscles. There was mild weakness of the iliopsoas and hamstrings and severe weakness of the tibialis anterior and peronei, more so on the left. The ankle jerks were just present with reinforcement. The other tendon reflexes were normal and plantar responses were flexor. There was a full range of low back movements and straight leg raising was not restricted. Sensation was normal save for a small area of decreased sensitivity on the left big toe. He walked with a left foot drop.

Blood count, erythrocyte sedimentation rate, biochemical profile, glucose, thyroid function tests, immunoglobulins, protein electrophoresis, and phytanic acid were all normal. There was a weakly positive antinuclear factor with a homogeneous pattern but there were no anti-MAG antibodies. DNA studies showed no duplication at $17 \mathrm{p} 11 \cdot 2$. A lumbar CT myelogram (fig 1c) showed thickening of all the nerve roots of the cauda equina, especially at the S1 level, where the exit foraminae were enlarged. No bony or disc lesions were seen except for L4-5 disc space narrowing. The CSF protein content was very high at $2 \cdot 2 \mathrm{~g} / \mathrm{l}$. Nerve conduction studies (table) indicated a mixed polyneuropathy. EMG of distal lower limb muscles showed large motor unit potentials with a decreased interference pattern, in keeping with chronic partial denervation and reinnervation. A left sural nerve biopsy showed reduced numbers of myelinated fibres, some clusters of small regenerating fibres, thinly myelinated fibres, and a few onion bulbs.

$\mathrm{He}$ was treated with $80 \mathrm{mg}$ prednisolone daily. An increase in strength was demonstrable by three months and nerve conduction studies showed improvement in most nerves by six months. A year after starting treatment he could play 18 holes of golf. Examination after a further year was normal apart from mild weakness of ankle dorsiflexion, more so on the left. His steroid dose was slowly reduced to $5 \mathrm{mg}$ on alternate days. Despite his clinical improvement MRI of the lumbar region performed 30 months after starting steroids showed almost identical findings to the initial CT myelogram.

In July 1992 he noticed left sided deafness. Brainstem auditory evoked responses showed a delayed wave I and MRI disclosed a tumour in the inner ear. The lesion was biopsied and was found to be an enlarged facial nerve. Postoperatively he had a complete left facial palsy. A facial nerve anastomosis was performed using a greater auricular nerve graft, which has resulted in substantial recovery of mobility, particularly in the lower half of the face.

\section{Discussion}

We have reported three patients who had symptoms suggestive of lumbar root disease, in whom subsequent findings fulfilled clinical, neurophysiological, and pathological criteria for CIDP. ${ }^{12}$ There was no family history of neuropathy or foot deformity and genetic markers for HMSN1a were absent. Nerve conduction studies in the children of patient 3 were normal. Further support for the diagnosis of CIDP comes from the remarkable response to steroids in patients 2 and 3 . In patient 1 the disease was so advanced that response to treatment has been only modest.

The first patient presented with a left L1-2 radiculopathy and back pain followed by features of a right C8 root lesion, for which a decompressive laminectomy was carried out. Patient 2 had proximal lower limb weakness, initially thought to be due to lumbar root disease. The third patient had several episodes of back pain and symptoms suggesting bilateral L2-3 root irritation before the development of a left foot drop.

Radiculopathy is an unusual presentation of CIDP. Symptoms of root disease were not recorded in three large series totalling 205 affected patients. ${ }^{1-3}$ It has, however, been stressed that a pattern of weakness which includes proximal as well as distal muscles is a distinctive feature of CIDP. ${ }^{3}$ This probably indicates painless root involvement and it is thus correct to use the term polyradiculoneuropathy when referring to CIDP, as is now commonly done. It also explains the appreciable increase of the CSF protein seen in our and other patients.

Nerve root enlargement in our patients was striking. It was radiologically evident in the lumbar region in all three and was most advanced in patient 1 . This patient also had massive cervical root hypertrophy, whereas the thoracic region was seemingly unaffected. Why the dorsal nerve roots should be spared is unclear, but it raises the possibility that nerve root thickening is in some way dependent on influences which arise in the limbs but not the trunk. At surgery the lumbosacral nerve roots were matted together so thickly that they filled the spinal canal and no CSF could be obtained.

By contrast, peripheral nerve thickening was not a feature in our patients except in patient 1 , in whom it was mild. This discrepancy between nerve root and peripheral nerve involvement was also found pathologically, although it was the reverse pattern from that reported by Matsuda et al. ${ }^{6}$ Sural nerve biopsy showed some cellular infiltration and a mixed picture of axonal loss and regeneration on the one hand and demyelination on the other. Whorls of Schwann cell cytoplasm were scanty. By comparison, such onion bulbs were plentiful in the biopsy of a posterior nerve root in patient 1 . It could thus be that nerve biopsy in CIDP might be unrewarding or even misleading. Dyck and colleagues ${ }^{1}$ have already pointed out that sural nerve biopsy in these circumstances is often not as helpful as might have been expected. More recent authors have underlined the fact that the nerve pathology in CIDP is relatively non-specific and that clinical and electrodiagnostic assessment usually suffices to make the diagnosis. ${ }^{13}$ 
How reliable are nerve conduction studies in these circumstances? It is accepted that motor conduction is slowed in CIDP, to the extent that this finding forms part of the mandatory criteria for the diagnosis of this condition. ${ }^{12}$ In our patients there seemed to be a gradient, the most severely affected clinically and radiologically (patient 1 ) having the slowest conduction velocities and the most obvious motor conduction block, whereas the least affected (patient 2) barely met the criteria laid down by Cornblath et al. ${ }^{12}$

Spine MRI is a valuable addition to the diagnostic armamentarium in CIDP. Enlarged spinal roots may be identified in patients being investigated for a demyelinating polyneuropathy or, as in our patients, may be discovered unsuspectingly when root disease is being considered. Even in the absence of nerve root thickening, abnormal enhancement of the cauda equina after intravenous gadolinium -DTPA may be demonstrable in $\mathrm{CIDP}^{14}$ as well as in acute inflammatory polyradiculoneuropathy. ${ }^{15}$ Other conditions which need to be considered in the differential diagnosis of nerve root hypertrophy are neurofibromatosis, HMSN, lymphoma, and amyloidosis. ${ }^{16}$ To date few patients with CIDP have undergone lumbar MRI and further studies are needed to determine whether a substantial proportion of patients have spinal nerve root enlargement or whether this is only a rare association.

Upper motor neuron signs or features of more widespread CNS dysfunction have been reported in a proportion of patients with CIDP. ${ }^{10}$ Long tract signs may be due to cord compression by enlarged nerve roots, as in our patient 1 . There is a previously reported clinically similar patient who improved after decompressive laminectomy. ${ }^{7}$ Alternatively, several patients have been described with multiple sclerosis-like plaques in the optic nerves, cerebral white matter, brainstem, cerebellum, and spinal cord, ${ }^{17-19}$ but our patients had no features suggestive of demyelination of the CNS.

Cranial nerve involvement occurs in about one in six patients with CIDP, ${ }^{1-3}$ probably caused by segmental demyelination. The most common finding is facial weakness followed by bulbar palsy, an eye movement disorder, and papilloedema. Patients with pronounced nerve root enlargement may also have cranial nerve hypertrophy, as in our patient 3, who developed neural deafness due to auditory nerve compression by an enlarged facial nerve. No such cranial nerve thickening has been reported on cranial MRI of patients with CIDP, ${ }^{2021}$ even in those with focal cranial nerve involvement. ${ }^{22}$ Schoene and colleagues ${ }^{17}$ described a patient whose clinical features included bilateral facial weakness and nerve deafness, in whom subsequent necropsy showed noticeable enlargement of cranial nerves 3-12. Cranial nerve enlargement may be mistaken for other pathology and biopsy should be avoided in these patients in view of the risk of damage such as that which occurred in our patient. Fortunately his facial palsy has recovered after nerve grafting.

We thank Professor PK Thomas for helpful advice on patient 2 and Dr J Gillespie for performing the CT and MRI.

1 Dyck PJ, Lais AC, Ohta M, et al. Chronic inflammatory polyradiculoneuropathy. Mayo Clin Proc 1975;621-37.

2 McCombe PA, Pollard JD, McLeod JG. Chronic inflammatory demyelinating polyradiculoneuropathy. A clinical and electrophysiological study of 92 cases. Brain 1987; 110:1617-30.

3 Barohn RJ, Kissel JT, Warmolts JR, Mendell JR. Chronic inflammatory demyelinating polyradiculoneuropathy. Clinical characteristics, course and recommendations for diagnostic criteria. Arch Neurol 1989;46:878-84.

4 Austin JH. Recurrent polyneuropathies and their corticosteroid treatment with five-year observations of a placebocontrolled case treated with corticotrophin, cortisone and prednisone. Brain 1958;81:157-94.

5 Harris W, Newcomb WD. A case of relapsing interstitial hypertrophic polyneuritis. Brain 1929;52:108-16.

6 Matsuda M, Sakurai S, Yanagisawa N. Hypertrophic neuritis due to chronic inflammatory demyelinating polyradiculoneuropathy (CIDP): a postmortem pathological culoneuropathy (CIDP): a postm

7 Symonds CP, Blackwood W. Spinal cord compression in hypertrophic neuritis. Brain 1958;85:251-60.

8 de Leon GA, Hodges FJ. Subarachnoid block and enlargement of the spinal canal in hypertrophic neuritis. $\mathcal{F}$ Neurol Sci $1976 ; 28: 139-46$.

9 Naganuma $M$, Doi S, Shima K, et al. Chronic inflammatory demyelinating polyradiculoneuropathy associated with multifocal nerve hypertrophy-report of a case with MRI study. Rinsho-Shinkeigaku 1991;31:1186-91.

10 De Silva R, Doyle D, Hadley D, et al. Nerve root hypertrophy in chronic inflammatory demyelinating polyneuropathy. Muscle Nerve 1994;17:168-70.

11 Ginsberg L, Platts AD, Thomas PK. Chronic inflammatory demyelinating polyneuropathy mimicking a lumbar spinal stenosis syndrome. 7 Neurol Neurosurg Psychiatry 1995;59:189-91.

12 Ad Hoc Subcommittee of the American Academy of Neurology AIDS Task Force. Research criteria for diagnosis of chronic inflammatory demyelinating polyneunosis of chronic inflammatory demyelinating

13 Krendel DA, Parks HP, Anthony DC, et al. Sural nerve biopsy in chronic inflammatory demyelinating polyradiculoneuropathy. Muscle Nerve 1989;12:257-64.

14 Crino PB, Grossman RI, Rostami A. Magnetic resonance imaging of the cauda equina in chronic inflammatory demyelinating polyneuropathy. Ann Neurol 1993;33: 311-3.

15 Morgan GW, Barohn RJ, Bazan III C, et al. Nerve root enhancement with MRI in inflammatory demyelinating polyradiculoneuropathy. Neurology 1993;43:618-9.

16 Antoine JC, Baril A, Guettier C, et al. Unusual amyloid polyneuropathy with predominant lumbosacral nerve roots and plexus involvement. Neurology 1991;41: roots $206-8$.

17 Schoene WC, Carpenter S, Behan, PO, Geschwind N. "Onion bulb" formations in the central and peripheral "Onion bulb" formations in the central and peripheral nervous system in association with multiple sclerosis an

18 Rosenberg NL, Bourdette D. Hypertrophic neuropathy and multiple sclerosis. Neurology 1983;33:1361-4

19 Thomas PK, Walker RWH, Rudge P, et al. Chronic demyelinating peripheral neuropathy associated with multifocal central nervous system demyelination. Brain 1987;110:53-76.

20 Ormerod IEC, Waddy HM, Kermode AG, et al. Involvement of the central nervous system in chronic inflammatory demyelinating polyneuropathy: a clinical, electrophysiological, and magnetic resonance imaging electrophysiological, and magnetic resonance imaging
study. $\mp$ Neurol Neurosurg Psychiatry 1990;53:789-93.

21 Hawke SHB, Hallinan JM, McLeod JG. Cranial magnetic resonance imaging in chronic demyelinating polyneuropathy. I Neurol Neurosurg Psychiatry 1990;53:794-6.

22 Waddy HM, Misra VP, King RHM. Focal cranial involvement in chronic inflammatory demyelinating polyneument in chronic inflammatory demyelinating polyneu-
ropthy: clinical and MRI evidence of peripheral and ropthy: clinical and MRI evidence of
central lesions. $₹$ Neurol 1989;236:400-5. 